\title{
BMJ Open Repetitive transcranial magnetic stimulation (rTMS) in autism spectrum disorder: protocol for a multicentre randomised controlled clinical trial
}

To cite: Enticott $P G$, Barlow K, Guastella AJ, et al. Repetitive transcranial magnetic stimulation (rTMS) in autism spectrum disorder: protocol for a multicentre randomised controlled clinical trial. BMJ Open 2021;11:e046830. doi:10.1136/ bmjopen-2020-046830

- Prepublication history and additional supplemental material for this paper are available online. To view these files, please visit the journal online. To view these files, please visit the journal online (http://dx.doi org/10.1136/bmjopen-2020046830).

Received 11 November 2020 Accepted 23 June 2021

Check for updates

(C) Author(s) (or their employer(s)) 2021. Re-use permitted under CC BY-NC. No commercial re-use. See rights and permissions. Published by BMJ.

For numbered affiliations see end of article.

Correspondence to Professor Peter G Enticott; peter.enticott@deakin.edu.au

\section{ABSTRACT}

Introduction There are no well-established biomedical treatments for the core symptoms of autism spectrum disorder (ASD). A small number of studies suggest that repetitive transcranial magnetic stimulation (rTMS), a non-invasive brain stimulation technique, may improve clinical and cognitive outcomes in ASD. We describe here the protocol for a funded multicentre randomised controlled clinical trial to investigate whether a course of rTMS to the right temporoparietal junction (rTPJ), which has demonstrated abnormal brain activation in ASD, can improve social communication in adolescents and young adults with ASD.

Methods and analysis This study will evaluate the safety and efficacy of a 4-week course of intermittent theta burst stimulation (iTBS, a variant of rTMS) in ASD. Participants meeting criteria for Diagnostic and Statistical Manual of Mental Disorders, Fifth Edition ASD ( $n=150$, aged $14-40$ years) will receive 20 sessions of either active iTBS (600 pulses) or sham iTBS (in which a sham coil mimics the sensation of iTBS, but no active stimulation is delivered) to the rTPJ. Participants will undergo a range of clinical, cognitive, epi/genetic, and neurophysiological assessments before and at multiple time points up to 6 months after iTBS. Safety will be assessed via a structured questionnaire and adverse event reporting. The study will be conducted from November 2020 to October 2024.

Ethics and dissemination The study was approved by the Human Research Ethics Committee of Monash Health (Melbourne, Australia) under Australia's National Mutual Acceptance scheme. The trial will be conducted according to Good Clinical Practice, and findings will be written up for scholarly publication.

Trial registration number Australian New Zealand Clinical Trials Registry (ACTRN12620000890932).

\section{Strengths and limitations of this study}

- This multisite randomised controlled trial will be the largest trial of repetitive transcranial magnetic stimulation (rTMS) in autism spectrum disorder (ASD) to date.

- rTMS will be applied to the right temporoparietal junction (rTPJ), a cortical region that has demonstrated abnormal activation in ASD and forms a major hub of the 'social brain' subnetwork.

- Participants will undergo structural MRI scans, with rTMS coil position determined via individualised neuronavigation.

- Adolescent and young adult participants will receive rTMS interventions as outpatients, and complete a comprehensive range of clinical, neuropsychological and neurophysiological assessments.

- A limitation of the study is the use of only a sham control condition, rather than an additional 'active control' site to determine whether effects are specific to rTPJ (rather than a general effect of brain stimulation).

\section{INTRODUCTION}

Autism spectrum disorder (ASD) is a neurodevelopmental disorder that impacts a range of domains, including social communication, behaviour, cognition, emotion regulation and sensorimotor function. ${ }^{1}$ Core symptoms of ASD include social interaction and communication problems, and restricted and repetitive behaviours. Comorbid neurodevelopmental disorders (eg, attention deficit hyperactivity disorder (ADHD)) and psychiatric disorders (eg, depression, anxiety) are 
very common, ${ }^{2}{ }^{3}$ with the latter often associated with the core social communicative difficulties. ${ }^{45}$

Despite the high prevalence of ASD (1 in 59), ${ }^{6}$ few clinical interventions target core symptoms beyond early-middle childhood. ASD diagnosis typically occurs by the age of $4-6$ years, ${ }^{6}$ and early, intensive intervention throughout these years is associated with the best outcomes for individuals with ASD and their families. ${ }^{7}$ Unfortunately, there is little clinical support available for adolescents and young adults with ASD, who often continue to experience social communication symptoms that result in barriers to education, employment and community participation. As noted, this group also experiences extremely poor mental health that is much worse than the general population; for instance, lifetime depression and anxiety rates are estimated at $37 \%$ and $42 \%$, respectively. $^{2}$

Non-invasive brain stimulation (NIBS) has emerged as a novel, safe and efficacious intervention for a range of brain-based disorders. These techniques allow noninvasive modulation of specific brain regions via electromagnetic or electrical stimulation. The most common of these is repetitive transcranial magnetic stimulation (rTMS), which is now widely used as an intervention for treatment-resistant major depressive disorder. ${ }^{8}$ It has also been established as an intervention for other neurological disorders, including migraine and obsessive-compulsive disorder. $^{9} 10$ rTMS is administered via a plastic-coated metallic coil that is held against the scalp. This coil emits focal, time-varying electromagnetic pulses, which induce electrical current in superficial cortical tissue, thus stimulating neurons in the local region. Depending on the frequency and strength of pulses administered, rTMS can be used to either enhance cortical excitability (ie, upregulate neural activity), or decrease cortical excitability (ie, downregulate neural activity) in the stimulated region. This is particularly useful when targeting regions (or nodes) of brain networks known to be either underactive or overactive in particular conditions. For instance, highfrequency (excitatory) rTMS has been used to stimulate underactive left dorsolateral prefrontal cortex (DLPFC) in treatment-resistant depression, ${ }^{11}$ while low-frequency (inhibitory) rTMS has been used to downregulate excessive activity in left auditory cortex in schizophrenia ${ }^{12}$ and supplementary motor area (SMA) in Tourette's disorder. ${ }^{13}$ Importantly, rTMS also influences broader brain networks that involve the stimulated region, ${ }^{14} 15$ and this is thought to contribute to its clinical efficacy. Here we will stimulate the right temporoparietal junction (rTPJ), a key node for social cognition, which is a typical area of difficulty among individuals with ASD. ${ }^{16}$

The brain functions as a set of interconnected networks disseminating neuronal information across a broad range of distributed areas. ${ }^{17}$ From a neurobiological perspective, ASD is commonly understood as a disorder of synaptic plasticity and neural connectivity, leading to abnormalities in brain network connectivity between brain regions. These appear to be mediated by disruptions in both excitatory (eg, glutamatergic) and inhibitory (eg, GABAergic) processes. ${ }^{18} 19$ There are also well-documented abnormalities in local 'node' activity, particularly within networks that comprise the so-called 'social brain,' including rTPJ. ${ }^{20} 21$ Indeed, the rTPJ shows consistent differences in activation between those with and without ASD, ${ }^{1620-22}$ while meta-analysis demonstrates reduced rTPJ functional connectivity in ASD. ${ }^{23}$ Accordingly, rTMS to this region has the potential to modulate local and regional brain activity within networks implicated in the core social symptoms of ASD.

rTMS is considered a very safe and tolerable technique. It is typically administered by an experienced clinician (nurse or physician), and patients are monitored throughout and at the completion of rTMS administration. Clinical researchers have established a detailed set of safety guidelines, and when rTMS is administered within guideline parameters, serious adverse effects are exceedingly rare. ${ }^{24}{ }^{25}$ NIBS (including rTMS) is also considered very safe for paediatric populations, with a recent study showing no adverse effects across 382 children aged $0-18$ years. ${ }^{26}$

Previously, NIBS has been used to investigate the neuropathophysiology of ASD. ${ }^{27-30}$ More recently, several research groups (including ours) have investigated whether rTMS could have clinical utility as an intervention in ASD. These studies (see systematic reviews ${ }^{31-33}$ ) indicate that: low-frequency stimulation of the DLPFC can reduce repetitive behaviours, improve neurophysiological markers of perception and reduce irritability; low-frequency SMA stimulation can improve movementrelated cortical potentials; and low-frequency stimulation of the premotor cortex can improve sensorimotor integration. While promising, these studies are hampered by small sample sizes, a lack of an appropriate control condition (placebo or sham stimulation), and moderateto-high risk of bias. ${ }^{31}$

At present, only two placebo-controlled randomised controlled trials (RCTs) have been conducted, both of which were double-blind. The first demonstrated that 2 weeks of daily, high-frequency rTMS to bilateral dorsomedial prefrontal cortex, compared with sham rTMS, improved self-report social-relating symptoms in adults with ASD $(n=28) 1$ month after intervention completion. $^{34}$ A recent study demonstrated that 4 weeks of high-frequency stimulation of bilateral DLPFC did not improve executive function in adolescents and young adults with ASD $(n=40) .{ }^{35}$ There was, however, evidence for a beneficial effect of rTMS for those with lower adaptive functioning at baseline. While providing preliminary, placebo-controlled support for rTMS in ASD, these studies are limited by small sample size.

\section{Rationale/justification}

A recent international 'consensus statement' provides recommendations for future rTMS research in ASD. ${ }^{36}$ Considering the clinical heterogeneity of ASD, there is agreement that 'large, multisite, double-blind, 
placebo-controlled trials with carefully selected neurobiological targets and outcome measures' are required. It is also necessary to understand variability in the response to rTMS that can lead to an individualised therapeutic approach (ie, personalised medicine approach). These include demographic (eg, age, sex), clinical (eg, disorder severity, cognitive/symptom profile), neurobiological (eg, cortical thickness, structural and functional connectivity), and genetic/epigenetic factors. Accordingly, we will conduct a large-scale, multisite investigation of the safety and efficacy of rTMS in ASD that involves (a) feasible and tolerable stimulation paradigms, (b) a carefully selected neurobiological target and mode of stimulation, and (c) rigorous methodological approaches, including individualised stereotactic neuronavigation, an appropriate control condition, and efficacious double blinding. If successful, this trial will establish a first biomedical intervention to improve social communicative symptoms in adolescents and young adults diagnosed with ASD, and inform on factors associated with intervention response, with anticipated benefits in mental health, quality of life and social participation.

\section{Research hypotheses}

In ASD, active rTMS to rTPJ, when compared with sham rTMS, will be associated with:

1. Improved social communication, measured using the Social Responsiveness Scale-Second Edition (SRS-2) (evident 1 month after end of rTMS, maintained at 3 and 6 months) (primary outcome).

2. Improved social cognitive performance, measured using face processing/face emotion processing neuropsychological tasks (evident immediately after rTMS, maintained at 1, 3 and 6 months).

3. Improved quality of life, measured using the Personal Wellbeing Index (PWI) (evident 1 month after rTMS, maintained at 3 and 6 months).

4. Acceptable tolerability and safety (as measured by a structured interview and adverse event reporting).

\section{METHODS AND ANALYSIS}

\section{Study design and participants}

This is a 4-year multicentre Australian study to assess the safety and efficacy of a 4-week course of rTMS to improve social communication in adolescents and young adults diagnosed with ASD. It will be a parallel group (between-subjects), double-blind, placebo-controlled RCT. Participants will be 150 individuals meeting criteria for Diagnostic and Statistical Manual of Mental Disorders, Fifth Edition (DSM-5) ${ }^{1}$ ASD and aged between 14 and 40 years. While broad, this age range was selected to ensure the feasibility of participant recruitment and to target age groups (ie, adolescents and young adults) where interventions for ASD are lacking. They will be recruited through existing research participant databases, the Australian Autism Biobank, ${ }^{37}$ and advertisements in local clinics, advocacy/support groups, and via social media.
The research team will also engage popular media, both locally and nationally, to promote recruitment.

The study will be overseen by a Research Management Group, which comprises the 10 chief investigators, study coordinator and site coordinators. They will meet monthly via video-conference for the duration of the trial. There will be 30 participants enrolled at each of the cities involved (Brisbane, Sydney, Melbourne, Adelaide, Perth). Participants will undergo 20 intervention sessions (one per weekday for 4 consecutive weeks) of either active or sham (ie, placebo) rTMS. Participants will be assessed before and up to 6 months after intervention and in accordance with Good Clinical Practice. Assessments will evaluate social communication, neuropsychological function, quality of life, safety and tolerability. There will be five primary intervention sites within Australia (Brisbane, Sydney, Melbourne, Adelaide, Perth) and additional local sites to support recruitment, assessment, genetic analysis, and neuroimaging. These will include both university and hospital sites. Written informed consent will be obtained from participants (or their parent/guardian in the case of minors, aged 14-17 years) by a local chief investigator or site coordinator. Model participant information and consent forms for parents/guardians and adult participants are provided as online supplemental material 2.

\section{Patient and public involvement}

The research team have engaged in extensive consultation with community groups in recent years, including multiple community forums on rTMS. We have also consulted with autism organisations when preparing advertisements and other study-related communications. While participants were not directly involved in the design of this specific trial, throughout the study we will engage a range of community and advocacy groups in the implementation of the research, and health service partners to ensure rapid translation of our research findings to clinical practice. For instance, the Telethon Kids Institute (Western Australia) has established a community reference group with whom they regularly consult for consumer involvement, and this group will also be engaged in the current trial.

\section{rTMS protocol}

Participants will receive standard intermittent theta burst stimulation (iTBS) to the rTPJ each consecutive weekday for a 4-week period (20 sessions). iTBS was chosen as it is an 'excitatory' paradigm that has the potential to target the reduced activation and connectivity commonly seen in rTPJ in ASD. ${ }^{16} 202123$ It can also be administered quickly and at a low intensity, which are important considerations in this clinical population. Participants will undergo either active iTBS or sham iTBS, where a 'sham coil' is used to mimic the appearance, sound and sensation of rTMS, but without delivering electromagnetic stimulation.

Participants will undergo 3T T1 MRI prior to the first rTMS session, and stereotactic neuronavigation will be used to determine the site of stimulation (Montreal 
56

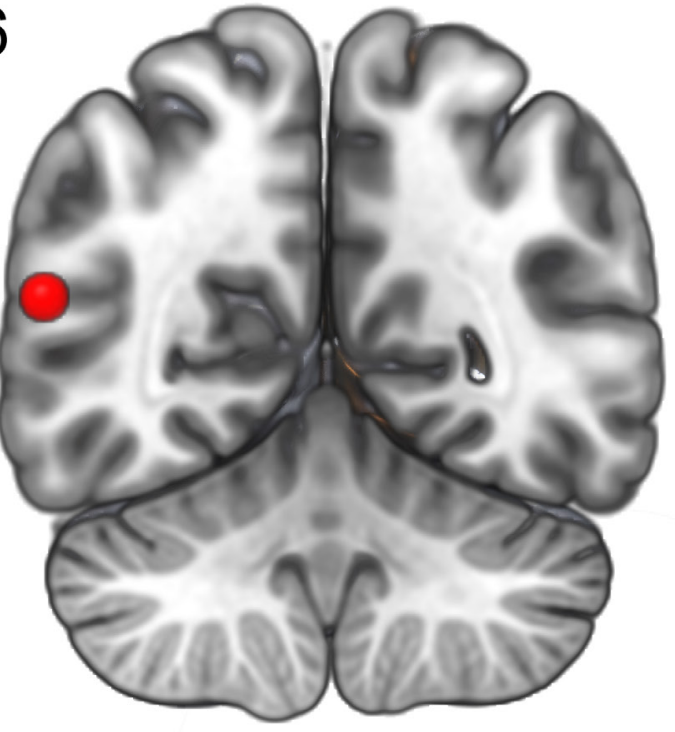

18

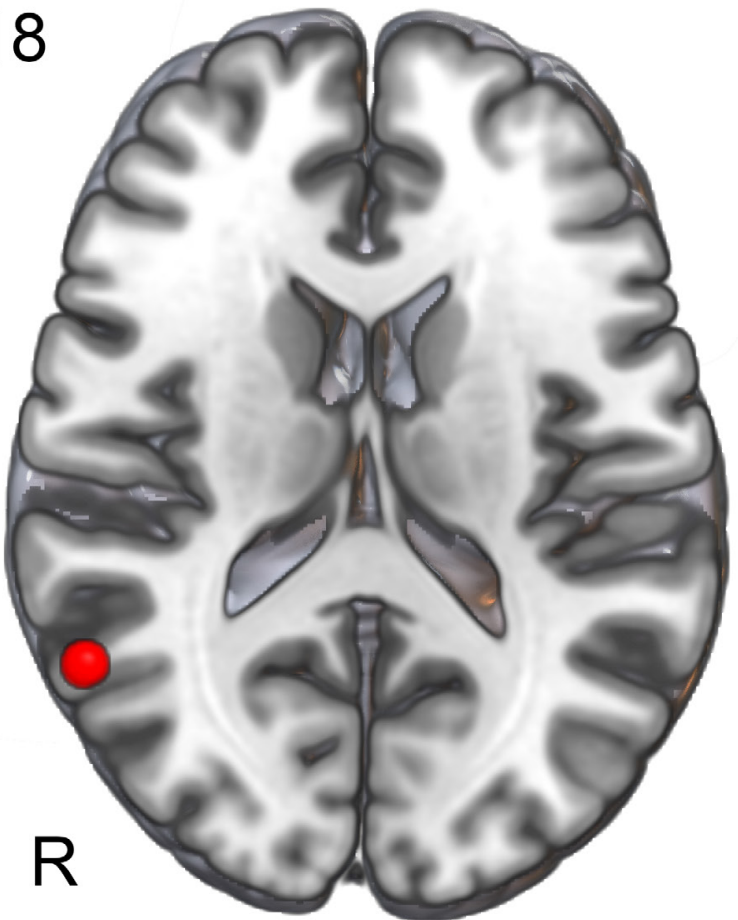

$-56$
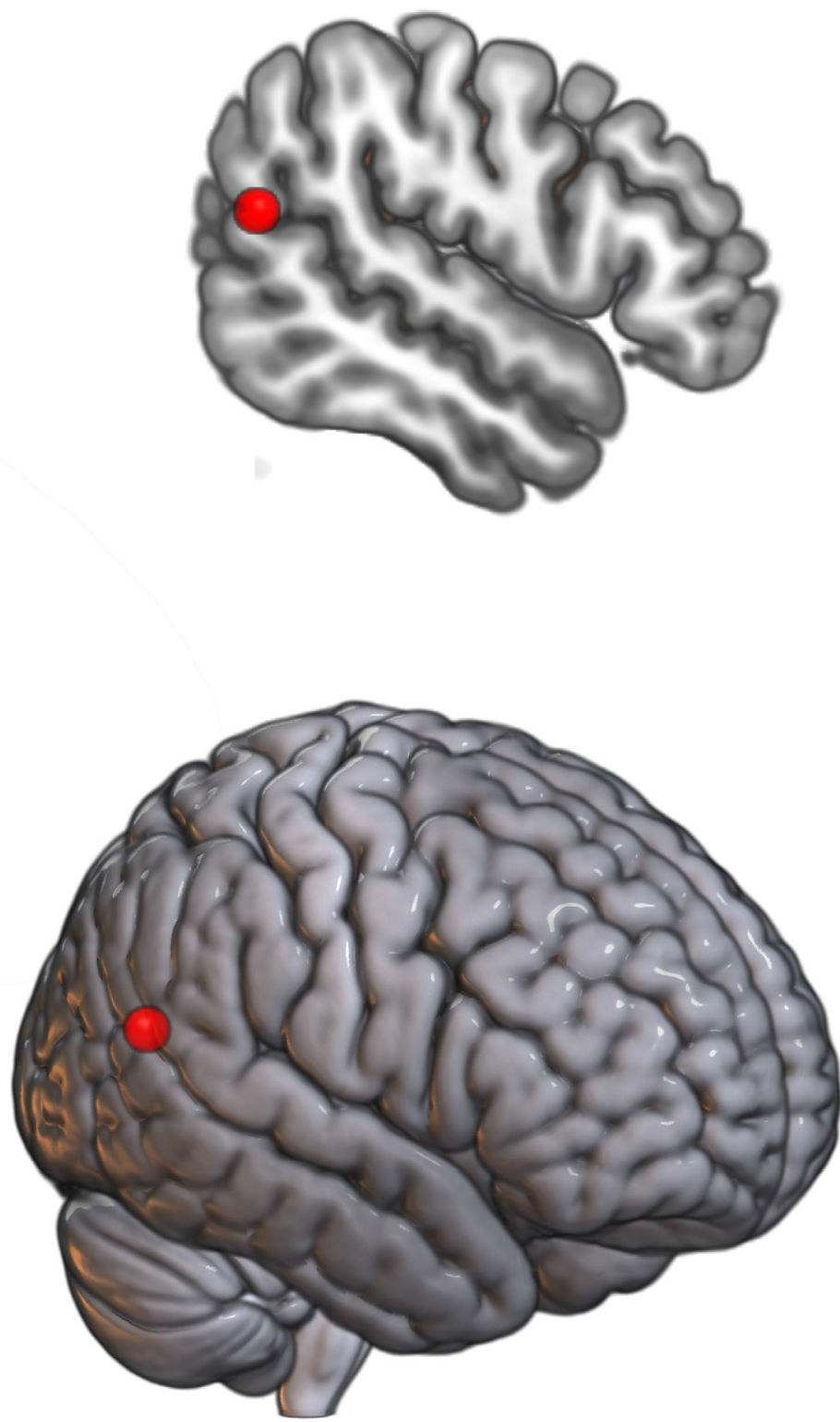

RA

Figure 1 Site of $r$ TMS coil localisation (MNI coordinates $x=56, y=-56, z=18$ ). MNI, Montreal Neurological Institute; rTMS, repetitive transcranial magnetic stimulation.

Neurological Institute coordinates $\mathrm{x}=56, \mathrm{y}=-56, \mathrm{z}=18$; see figure 1).

All stimulation will be administered via a Magstim Rapid $^{2}$ stimulator (The Magstim Company, Wales, UK). A staff member trained in rTMS will deliver all rTMS interventions. A visual resting motor threshold (ie, visual observation of muscle activation following TMS pulse) will be determined at the right hemisphere/left hand prior to the first rTMS session. Each iTBS is delivered with the following stimulation parameters:

- Burst pattern: three pulses delivered at $50 \mathrm{~Hz}$.
- Train duration: bursts repeated five times per second ( $5 \mathrm{~Hz})$ for $2 \mathrm{~s} \mathrm{(10} \mathrm{bursts).}$

- Intensity: $70 \%$ of resting motor threshold.

- Intertrain interval: $8 \mathrm{~s}$.

- Total time: $200 \mathrm{~s}$ (3 min, 20s).

- Total trains: 20.

- Total bursts: 200 .

- Total pulses: 600 .

All rTMS procedures, including resting motor threshold, will be administered by a TMS clinician who is not blinded to study condition. Participants will be 
monitored by study staff for at least 5 min after each intervention session. They can then leave the facility and go about their normal daily activities, including driving. The participant will be administered the non-invasive brain stimulation post-stimulation interview at the end of each week of rTMS intervention (ie, after the Friday session) to determine the presence/intensity of any side effects. For child participants (aged 14-17 years), this interview will be conducted with both the parent/guardian and the child. Participants will also be regularly asked about their well-being during and immediately after each rTMS session. Any side effects reported in this manner will be documented in the participant's file and will be examined at the completion of the trial.

\section{Inclusion and exclusion criteria}

Inclusion criteria

- Aged 14-40 years.

- Meets criteria for ASD based on DSM-5 criteria (clinician reported), and confirmed via Autism Diagnostic Observation Schedule-Second Edition (ADOS-2).

- English-language fluency/proficiency.

Exclusion criteria

- History of seizure/s or epilepsy.

- History of severe (traumatic) brain injury.

- Contraindication to MRI (eg, claustrophobia, metal implants).

- Formal verbal IQ assessment $<55$, as determined by Wechsler Abbreviated Scale of Intelligence.

- Comorbid neurological or psychiatric diagnosis not commonly associated with ASD (eg, psychosis).

- Unstable medical condition.

- Unstable medication regimen, or medication contraindicated for TMS.

- Pregnancy or current breast feeding.

- Substance use/abuse disorder.

- Concurrent intervention targeting social communication.

- Evidence of significant epileptiform activity on electroencephalogram (EEG) (eg, seizures on EEG, runs of epileptiform discharges).

\section{Outcome measures}

Data collection and study timings are presented in table 1. Participants are assessed prior to rTMS (T0), and at four points after rTMS: T1 (immediately after rTMS), T2 (1 month after completion of rTMS), T3 (3 months after completion of rTMS) and T4 (6 months after completion of rTMS).

The primary outcome measure is the SRS-2 (school-age autoscore form for parent/guardian (parent/guardian report)/adult autoscore form for informant (informant report)) total T-score, while the primary outcome point will be at 1 month after completion of rTMS (T2) compared with pre-rTMS (T0). For adult participants, an informant (parent/relative/friend) will complete the SRS-2 with respect to the participant.
Secondary outcomes encompass a range of clinical, neuropsychological, neurophysiological and biological measures. Clinical measures include: Conners 3 (parent/guardian report)/Conners Adult ADHD Rating Scales (informant report and adult self-report); Aberrant Behaviour Checklist-Second Edition (parent/ guardian/informant report); Behaviour Rating Scale of Executive Function, Second Edition (BRIEF)/BRIEFAdult Version (parent/guardian/informant report and adult self-report); WHO Disability Assessment Schedule 2.0 (parent/guardian/informant report); Depression, Anxiety and Stress Scale (self-report); and PWI (self-report).

Neuropsychological measures include: Reading the Mind in the Eyes Test; Benton Facial Recognition Test; Cambridge Face Memory Test; National Institutes of Health Cognition Toolbox; and Working Memory Assessment.

Neurophysiological measures include: resting-state EEG and face processing event-related potentials.

The neuropsychological and neurophysiological measures were selected as they are associated with activation of the target cortical region $\left(\mathrm{eg},{ }^{38}\right)$; while there were additional paradigms that could have been used (eg, biological motion processing), we were mindful of not overburdening our participants and selected those that we felt most relevant to our social communication target.

Finally, a buccal swab will be administered both before and after the course of rTMS, which will allow an investigation of genetic and epigenetic predictors of intervention response, and potential epigenetic changes following rTMS.

The various electrophysiological and genetic measures that are being collected are highly exploratory but may help us to understand mechanisms by which rTMS exerts an influence on social communication.

\section{Randomisation}

There will be an equal number of participants allocated to each condition at each of the five project sites (15 active, 15 sham; total 75 active, 75 sham). A computerised adaptive randomisation procedure (minimisation method) will be performed, adjusting for baseline characteristics (age, sex, SRS T0 score), ${ }^{39}$ which ensures a balance of conditions across trial sites. Randomisation will be completed by the chief investigator, who will provide this information to the intervention clinicians (who are not blinded) via email.

\section{Statistical analysis and data management}

With respect to statistical power, allowing for $10 \%$ attrition of our 150 participants, and based on the estimated effect size from our previously published RCT (which revealed a moderate effect of rTMS), ${ }^{34}$ a sample size of $n=135$ in a mixed-model (two groups, five time points) will yield power of $0.99(\mathrm{f}=0.20, \alpha=0.01)$. While this sample size is larger than the minimum suggested by a priori power analysis $(n=64$, based on $\mathrm{f}=0.20, \alpha=0.01$, power $=0.95)$, 
Table 1 Data collection and study timings

\begin{tabular}{|c|c|c|c|c|c|c|c|}
\hline Visits & Pre-enrolment & TO & Tx & T1 & T2 & T3 & T4 \\
\hline Screening & $x$ & $x$ & & & & & \\
\hline Written informed consent & & $x$ & & & & & \\
\hline Randomisation & & $x$ & & & & & \\
\hline Demographics & $x$ & $x$ & & & & & \\
\hline Medical history & $x$ & $x$ & & & & & \\
\hline Neuroimaging (MRI) & & $x$ & & & & & \\
\hline Clinical EEG & & $x$ & & & & & \\
\hline Buccal swab & & $x$ & & $x$ & & & \\
\hline ADOS-2 & & $x$ & & & & & \\
\hline WASI-2 & & $x$ & & & & & \\
\hline rTMS intervention (active/sham) & & & $x$ & & & & \\
\hline NIBS:PSI & & & $x$ & & & & \\
\hline SRS-2 & & $x$ & & $x$ & $x$ & $x$ & $x$ \\
\hline Conners-3/CAARS & & $x$ & & $\mathrm{x}$ & $x$ & $x$ & $x$ \\
\hline$A B C-2$ & & $x$ & & $x$ & $x$ & $x$ & $x$ \\
\hline BRIEF/BRIEF-A & & $x$ & & $x$ & $\mathrm{X}$ & $x$ & $\mathrm{x}$ \\
\hline DASS & & $x$ & & $x$ & $x$ & $x$ & $x$ \\
\hline PWI & & $x$ & & $\mathrm{x}$ & $\mathrm{x}$ & $x$ & $x$ \\
\hline WHODAS 2.0 & & $x$ & & $x$ & $x$ & $x$ & $x$ \\
\hline NIH Cognition Toolbox & & $x$ & & $\mathrm{x}$ & $x$ & & $x$ \\
\hline RMET & & $x$ & & $x$ & $x$ & & $x$ \\
\hline BFRT & & $x$ & & $\mathrm{x}$ & $x$ & & $x$ \\
\hline CFMT & & $x$ & & $x$ & $x$ & & $x$ \\
\hline Working Memory & & $x$ & & $x$ & $x$ & & $x$ \\
\hline rsEEG & & $x$ & & $x$ & & & \\
\hline FP-ERP & & $x$ & & $X$ & & & \\
\hline
\end{tabular}

ABC-2, Aberrant Behaviour Checklist, Second Edition; ADHD, attention deficit hyperactivity disorder; ADOS-2, Autism Diagnostic Observation Schedule, Second Edition; BFRT, Benton Facial Recognition Test; BRIEF, Brief Rating Inventory of Executive Function; BRIEF-A, BRIEF-Adult Version; CAARS, Conners Adult ADHD Rating Scales; CFMT, Cambridge Face Memory Test; DASS, Depression, Anxiety and Stress Scale; EEG, electroencephalography; FP-ERP, face-processing event-related potentials; NIBS:PSI, non-invasive brain stimulation post-stimulation interview; NIH, National Institutes of Health; PWI, Personal Wellbeing Index; RMET, Reading the Mind in the Eyes Test; rsEEG, resting-state EEG; rTMS, repetitive transcranial magnetic stimulation; SRS-2, Social Responsiveness Scale, Second Edition; T0, pre-rTMS; T1, week following rTMS; T2, 1 month after completion of rTMS; T3, 3 months after completion of rTMS; T4, 6 months after completion of rTMS; WASI2, Wechsler Abbreviated Scale of Intelligence, Second Edition; WHODAS 2.0, WHO Disability Assessment Schedule.

this will enable exploratory analysis to determine demographic, clinical, neuroimaging and genetic predictors of treatment response.

On enrolment, participants will be allocated a unique study identification code. Their name will not appear with the research data collected. All data will be stored in REDCap $^{40}$ and on secure network locations governed by Deakin University. All chief investigators will have access to the final trial dataset. Any information obtained in connection with this research project that can identify a participant will remain confidential. Where a participant elects to withdraw from the study, we will retain and use any data collected prior to withdrawal.

Random effects linear mixed models will be used to ensure the inclusion of participants who have missing data, including those that withdraw from the study. Specifically, this will involve a between-subjects factor (rTMS condition: active vs placebo) and a within-subjects factor (time of assessment: pre vs post vs 1 month vs 3 months vs 6 months), with participant and site entered as random effects. We will employ an intention-to-treat framework for these analyses. We will examine rTMS safety by exploring descriptive statistics arising from the structured questionnaire related to the development of possible side effects. An interim analysis will be performed at the midpoint of data collection for possible trial futility. The above-mentioned a priori power analysis, where $n=64$ is required to detect a moderate effect, suggests that we will be sufficiently powered to detect an effect of rTMS in this interim analysis. 
Exploratory analyses will be undertaken to investigate factors, including genetic variants and structural/functional neuroimaging (eg, diffusion MRI, resting-state functional MRI), that influence intervention response, and to investigate epigenetic changes following rTMS. We will use linear mixed models to determine the effect of rTMS on SRS-2 score, but with additional independent variables (eg, age, sex, cognitive ability, ADOS-2 symptom severity, rTPJ structural and functional connectivity within the social brain subnetwork, polygenic risk score for $\left.\mathrm{ASD}^{41}\right)$.

Epigenetic variation refers to variation in chromatin structure, which is associated with variation in gene expression. In contrast to DNA, epigenetic variation can change over time, for example, following treatment. ${ }^{42}$ Accordingly, we will compare epigenetic variation for DNA samples collected before and after rTMS and investigate any associations with intervention response (see online supplemental material 1 for a statement on biological specimens).

At the conclusion of the project, all electronic and hard copy data will be archived within Deakin University (Information and Records Services). Electronic data will be retained on secure Deakin University servers and archived in REDCap, but also transferred to physical hard drives for archival storage. As some hard copy data will be stored at each site (eg, signed consent forms, clinical files used during rTMS intervention), these will be securely couriered to Deakin University for archiving. Each site will be required to delete any electronic data that may remain at their site. As this is a clinical trial involving child participants, data will be retained indefinitely. Any published work from this study will be accompanied by publicly available deidentified data through the Open Science Framework (osf.io). The research team, including both chief investigators and associate investigators, all have the opportunity to conduct secondary analyses. This will be negotiated with the trial's Research Management Group, which comprises the 10 chief investigators. Data may also be shared with external (national and international) collaborators to obtain larger sample sizes, which are often necessary to achieve the statistical power necessary to analyse biomarker data. This could include specific research projects or online data repositories, which may be accessed and used by external researchers.

\section{Blinding}

This is a double-blind study; accordingly, participants (and their parents/guardians, where relevant) and the testing researchers/statisticians will be blinded to intervention condition. The individual administering rTMS must select the appropriate coil (ie, active or sham) and will therefore not be blinded, but this individual will not conduct any of the assessment or be involved in the statistical analyses. Unblinding may occur in the event of an adverse event. We will assess blinding integrity by asking participants to indicate, at the end of their 4-week intervention, which condition they believed they received and the confidence (on an 11-point scale) in this judgement. At the conclusion of the final assessment (T4), participants will be unblinded as to their intervention condition by a member of the research team who is not blinded. Those who were allocated to the sham rTMS intervention will be offered the opportunity to undergo the real rTMS intervention. While this will occur after all assessments have been administered, scored and entered into REDCap, we have developed standard operating procedures to minimise the likelihood that assessors will encounter participants completing the open-label component.

\section{Safety}

Participants will undergo extensive screening to ensure that they meet safety criteria for undergoing rTMS. ${ }^{25}$ For child participants, a parent or legal guardian will complete the screening. Participants will undergo EEG prior to their first rTMS session, and this will be reviewed by the trial neurologist. Any participants demonstrating evidence of runs of epileptiform discharges, as assessed by the study neurologist, will be withdrawn from the study. At the beginning of their first session, participants (or their parent/guardian for child participants) will again be screened to ensure that they can undergo rTMS.

A Data Safety Monitoring Board (DSMB) will be formed. This DSMB will comprise three senior clinical researchers independent to the current project. The DSMB will meet twice per year to review the conduct of the trial and monitor study data. They will also review any serious adverse events in a mid-trial safety analysis and on an ad hoc basis. Terms of reference will be based on advice from the National Health and Medical Research Council's DSMB documentation.

Adverse events will be reported to the relevant Human Research Ethics Committees (HRECs) immediately, and no later than 72 hours after the event. Depending on the nature and severity of the event, it may be necessary to also report to other regulatory bodies (eg, Therapeutic Goods Administration) and suspend or terminate the trial. Should an individual suffer harm from trial participation, they will receive medical treatment required to treat the injury or complication, free of charge, as a public patient in any Australian public hospital.

\section{Ethics and dissemination}

This study has been approved by the Monash Health HREC (Melbourne, Australia; RES-20-0000-606A) under the National Mutual Acceptance scheme, which allows for mutual scientific and ethical acceptance across Australian jurisdictions and institutions. We will engage a range of community and advocacy groups in the implementation of the study, and health service partners to ensure rapid translation of our research findings to clinical practice.

The health outcomes of this study will be provided within 12 months of the trial's completion, initially through a freely accessible preprint and an open-access peer-reviewed journal publication. Authorship will be determined according to the standards outlined in the 
National Health and Medical Research Council's Australian Code for the Responsible Conduct of Research. Chief investigators will also present the study findings at relevant scientific conferences and autism advocacy/support group community forums. The research team will also engage in more extensive public outreach and disseminate study findings widely through appropriate channels (eg, study website, social media, news outlets). These dissemination pathways will also involve contributing to clinical guidelines (and direct engagement with healthcare providers).

Participants will be sent a plain language summary detailing the study results at the completion of the trial. This summary will be written as a lay summary and in a manner accessible to participants and their families. A child version will also be sent to parents/guardians to share with their child. The summary will contain no identifying information and provide only group-level results.

This project involves the collection of a large number of measures (eg, clinical, neuropsychological, neuroimaging, genetic/epigenetic) and it is expected that the chief investigators will conduct further exploratory analyses on these data. This might include, for example, examining neuroimaging and genetic predictors of response to rTMS intervention and characterising epigenetic changes following rTMS.

\section{Trial status}

At the time of submission, recruitment has not commenced.

\section{Author affiliations}

${ }^{1}$ School of Psychology, Deakin University, Geelong, Victoria, Australia

${ }^{2}$ Central Clinical School, Monash University, Melbourne, Victoria, Australia

${ }^{3}$ Child Health Research Centre, The University of Queensland, South Brisbane,

Queensland, Australia

${ }^{4}$ Children's Health Queensland Hospital and Health Service, South Brisbane,

Queensland, Australia

${ }^{5}$ Autism Clinic for Translational Research, Brain and Mind Centre, Children's Hospital Westmead Clinical School, Faculty of Medicine and Health, The University of Sydney,

Camperdown, New South Wales, Australia

${ }^{6}$ Telethon Kids Institute, Perth, Western Australia, Australia

${ }^{7}$ University of Western Australia, Crawley, Western Australia, Australia

${ }^{8}$ Discipline of Psychiatry, The University of Adelaide, Adelaide, South Australia, Australia

${ }^{9}$ Hopwood Centre for Neurobiology, Lifelong Health Theme, South Australian Health and Medical Research Institute, Adelaide, South Australia, Australia

${ }^{10}$ Turner Institute for Brain and Mental Health, School of Psychological Sciences and Monash Biomedical Imaging, Monash University, Melbourne, Victoria,

Australia

${ }^{11}$ Centre for Molecular Medicine and Innovative Therapeutics, Murdoch University, Murdoch, Western Australia, Australia

${ }^{12}$ Discipline of Psychology, Murdoch University, Murdoch, Western Australia,

Australia

${ }^{13}$ Brain and Mind Centre, Central Clinical School, Faculty of Medicine and Health, The University of Sydney, Camperdown, New South Wales, Australia

${ }^{14}$ IMPACT - the Institute for Mental and Physical Health and Clinical Translation, School of Medicine, Deakin University, Geelong, Victoria, Australia

${ }^{15}$ Krongold Clinic, Monash Education, Monash University, Clayton, Victoria, Australia

${ }^{16}$ Centre for Human Psychopharmacology, Faculty of Heath, Arts and Design,

Swinburne University of Technology, Melbourne, Victoria, Australia

${ }^{17}$ Epworth Centre for Innovation in Mental Health, Epworth HealthCare, Camperwell, Victoria, Australia
Contributors PGE, KB, AJG, ML, NR, CM, SC, A-MV, KB, IBH, CG, HF, HH, JC, MK, NM, PD and PF contributed to the design of the study. PGE, KB, ML, NR, CM, SC, A-MV, KB, AJOW, GA, MK, PD, TF, KC, NA-U, SB and PF contributed to the writing of the manuscript. All authors approved the final draft of the manuscript.

Funding This project has been funded by the National Health and Medical Research Council (NHMRC) under the Medical Research Future Fund (MRFF) Neurological Disorders 2020 scheme (Application ID: APP1199298).

Competing interests None declared.

Patient consent for publication Not required.

Provenance and peer review Not commissioned; externally peer reviewed.

Supplemental material This content has been supplied by the author(s). It has not been vetted by BMJ Publishing Group Limited (BMJ) and may not have been peer-reviewed. Any opinions or recommendations discussed are solely those of the author(s) and are not endorsed by BMJ. BMJ disclaims all liability and responsibility arising from any reliance placed on the content. Where the content includes any translated material, BMJ does not warrant the accuracy and reliability of the translations (including but not limited to local regulations, clinical guidelines, terminology, drug names and drug dosages), and is not responsible for any error and/or omissions arising from translation and adaptation or otherwise.

Open access This is an open access article distributed in accordance with the Creative Commons Attribution Non Commercial (CC BY-NC 4.0) license, which permits others to distribute, remix, adapt, build upon this work non-commercially, and license their derivative works on different terms, provided the original work is properly cited, appropriate credit is given, any changes made indicated, and the use is non-commercial. See: http://creativecommons.org/licenses/by-nc/4.0/.

\section{ORCID iDs}

Peter G Enticott http://orcid.org/0000-0002-6638-951X

Karen Barlow http://orcid.org/0000-0003-2612-8507

Adam J Guastella http://orcid.org/0000-0001-8178-4625

Melissa K Licari http://orcid.org/0000-0003-3705-5323

Nigel C Rogasch http://orcid.org/0000-0002-4484-1069

Christel M Middeldorp http://orcid.org/0000-0002-6218-0428

Ann-Maree Vallence http://orcid.org/0000-0001-9190-6366

Kelsie A Boulton http://orcid.org/0000-0002-9408-7367

lan B Hickie http://orcid.org/0000-0001-8832-9895

Cherrie Galletly http://orcid.org/0000-0001-6185-9677

Gail A Alvares http://orcid.org/0000-0003-3351-5919

Hakuei Fujiyama http://orcid.org/0000-0002-7546-6636

Helen Heussler http://orcid.org/0000-0003-0666-7810

Jeffrey M Craig http://orcid.org/0000-0003-3979-7849

Melissa Kirkovski http://orcid.org/0000-0003-3395-8525

Natalie T Mills http://orcid.org/0000-0003-3255-5118

Nicole J Rinehart http://orcid.org/0000-0001-6109-3958

Peter H Donaldson http://orcid.org/0000-0001-7715-8891

Talitha C Ford http://orcid.org/0000-0001-5400-2659

Karen Caeyenberghs http://orcid.org/0000-0001-7009-6843

Natalia Albein-Urios http://orcid.org/0000-0001-7841-018X

Soukayna Bekkali http://orcid.org/0000-0002-8055-0365

Paul B Fitzgerald http://orcid.org/0000-0003-4217-8096

\section{REFERENCES}

1 American Psychiatric Association. Diagnostic and statistical manual of mental disorders. 5th ed. Arlington, VA: American Psychiatric Association, 2013.

2 Hollocks MJ, Lerh JW, Magiati I, et al. Anxiety and depression in adults with autism spectrum disorder: a systematic review and metaanalysis. Psychol Med 2019;49:559-72.

3 Rau S, Skapek MF, Tiplady K, et al. Identifying comorbid ADHD in autism: attending to the inattentive presentation. Res Autism Spectr Disord 2020;69:101468.

4 Day TC, McNaughton KA, Naples AJ, et al. Self-Reported social impairments predict depressive disorder in adults with autism spectrum disorder. Autism 2020;24:297-306.

5 van Steensel FJA, Bögels SM, Perrin S. Anxiety disorders in children and adolescents with autistic spectrum disorders: a meta-analysis. Clin Child Fam Psychol Rev 2011;14:302-17.

6 Baio J, Wiggins L, Christensen DL, et al. Prevalence of autism spectrum disorder among children aged 8 years - autism and 
developmental disabilities monitoring network, 11 sites, United States, 2014. MMWR Surveill. Summ. 2018;67:1-23.

7 Landa RJ. Efficacy of early interventions for infants and young children with, and at risk for, autism spectrum disorders. Int Rev Psychiatry 2018;30:25-39.

8 George MS, Lisanby SH, Avery D, et al. Daily left prefrontal transcranial magnetic stimulation therapy for major depressive disorder: a sham-controlled randomized trial. Arch Gen Psychiatry 2010;67:507-16.

9 Feng Y, Zhang B, Zhang J, et al. Effects of non-invasive brain stimulation on headache intensity and frequency of headache attacks in patients with migraine: a systematic review and metaanalysis. Headache 2019;59:1436-47.

10 Rapinesi C, Kotzalidis GD, Ferracuti S, et al. Brain stimulation in obsessive-compulsive disorder (OCD): a systematic review. Curr Neuropharmacol 2019;17:787-807.

11 Luber BM, Davis S, Bernhardt E, et al. Using neuroimaging to individualize TMS treatment for depression: toward a new paradigm for imaging-guided intervention. Neuroimage 2017;148:1-7.

12 Blumberger DM, Fitzgerald PB, Mulsant BH, et al. Repetitive transcranial magnetic stimulation for refractory symptoms in schizophrenia. Curr Opin Psychiatry 2010;23:85-90.

13 Hsu C-W, Wang L-J, Lin P-Y. Efficacy of repetitive transcranial magnetic stimulation for Tourette syndrome: a systematic review and meta-analysis. Brain Stimul 2018;11:1110-8.

14 Tik M, Hoffmann A, Sladky R, et al. Towards understanding rTMS mechanism of action: stimulation of the DLPFC causes network-specific increase in functional connectivity. Neuroimage 2017;162:289-96.

15 Caeyenberghs K, Duprat R, Leemans A, et al. Accelerated intermittent theta burst stimulation in major depression induces decreases in modularity: a connectome analysis. Netw Neurosci 2019;3:157-72.

16 Lombardo MV, Chakrabarti B, Bullmore ET, et al. Specialization of right temporo-parietal junction for mentalizing and its relation to social impairments in autism. Neuroimage 2011;56:1832-8.

17 Bullmore E, Sporns O. The economy of brain network organization. Nat Rev Neurosci 2012;13:336-49.

18 Coghlan S, Horder J, Inkster B, et al. Gaba system dysfunction in autism and related disorders: from synapse to symptoms. Neurosci Biobehav Rev 2012;36:2044-55.

19 Hull JV, Dokovna LB, Jacokes ZJ, et al. Resting-State functional connectivity in autism spectrum disorders: a review. Front Psychiatry 2016;7:205.

20 Patriquin MA, DeRamus T, Libero LE, et al. Neuroanatomical and neurofunctional markers of social cognition in autism spectrum disorder. Hum Brain Mapp 2016;37:3957-78.

21 Philip RCM, Dauvermann MR, Whalley HC, et al. A systematic review and meta-analysis of the fMRI investigation of autism spectrum disorders. Neurosci Biobehav Rev 2012;36:901-42.

22 Kirkovski M, Enticott PG, Hughes ME, et al. Atypical neural activity in males but not females with autism spectrum disorder. J Autism Dev Disord 2016:46:954-63.

23 Wang W, Liu J, Shi S, et al. Altered resting-state functional activity in patients with autism spectrum disorder: a quantitative meta-analysis. Front Neurol 2018;9:556.

24 Lerner AJ, Wassermann EM, Tamir DI. Seizures from transcranial magnetic stimulation 2012-2016: results of a survey of active laboratories and clinics. Clin Neurophysiol 2019;130:1409-16.

25 Rossi S, Hallett M, Rossini PM, et al. Safety, ethical considerations, and application guidelines for the use of transcranial magnetic stimulation in clinical practice and research. Clin Neurophysiol 2009;120:2008-39.

26 Zewdie E, Ciechanski P, Kuo HC, et al. Safety and tolerability of transcranial magnetic and direct current stimulation in children: prospective single center evidence from 3.5 million stimulations. Brain Stimul 2020;13:565-575.

27 Cole EJ, Barraclough NE, Enticott PG. Investigating mirror system (MS) activity in adults with ASD when inferring others' intentions using both TMS and EEG. J Autism Dev Disord 2018;48:2350-67.

28 Enticott PG, Kennedy HA, Rinehart NJ, et al. Gabaergic activity in autism spectrum disorders: an investigation of cortical inhibition via transcranial magnetic stimulation. Neuropharmacology 2013;68:202-9.

29 Enticott PG, Kennedy HA, Rinehart NJ, et al. Mirror neuron activity associated with social impairments but not age in autism spectrum disorder. Biol Psychiatry 2012;71:427-33.

30 Kirkovski M, Rogasch NC, Saeki T, et al. Single pulse transcranial magnetic Stimulation-Electroencephalogram reveals no electrophysiological abnormality in adults with high-functioning autism spectrum disorder. J Child Adolesc Psychopharmacol 2016;26:606-16.

31 Barahona-Corrêa JB, Velosa A, Chainho A, et al. Repetitive transcranial magnetic stimulation for treatment of autism spectrum disorder: a systematic review and meta-analysis. Front Integr Neurosci 2018;12:27.

32 Enticott PG, Kirkovski M, Oberman LM. Transcranial magnetic stimulation in autism spectrum disorder. In: Oberman LM, Enticott PG, eds. Neurotechnology and brain stimulation in pediatric psychiatric and neurodevelopmental disorders. London, UK: Elsevier, 2019.

33 Oberman LM, Enticott PG, Casanova MF, et al. Transcranial magnetic stimulation in autism spectrum disorder: challenges, promise, and roadmap for future research. Autism Res 2016;9:184-203.

34 Enticott PG, Fitzgibbon BM, Kennedy HA, et al. A double-blind, randomized trial of deep repetitive transcranial magnetic stimulation (rTMS) for autism spectrum disorder. Brain Stimul 2014;7:206-11.

35 Ameis SH, Blumberger DM, Croarkin PE, et al. Treatment of executive function deficits in autism spectrum disorder with repetitive transcranial magnetic stimulation: a double-blind, sham-controlled, pilot trial. Brain Stimul 2020;13:539-47.

36 Cole EJ, Enticott PG, Oberman LM, et al. The potential of repetitive transcranial magnetic stimulation for autism spectrum disorder: a consensus statement. Biol Psychiatry 2019;85:e21-2.

37 Alvares GA, Dawson PA, Dissanayake C, et al. Study protocol for the Australian autism Biobank: an international resource to advance autism discovery research. BMC Pediatr 2018;18:284.

38 Gao C, Conte S, Richards JE, et al. The neural sources of N170: understanding timing of activation in face-selective areas. Psychophysiology 2019;56:e13336-e36.

39 Kenjo Y, Antoku Y, Akazawa K, et al. An easily customized, random allocation system using the minimization method for multiinstitutional clinical trials. Comput Methods Programs Biomed 2000;62:45-9.

40 Harris PA, Taylor R, Minor BL, et al. The REDCap Consortium: building an international community of software platform partners. $J$ Biomed Inform 2019;95:103208.

41 Grove J, Ripke S, Als TD, et al. Identification of common genetic risk variants for autism spectrum disorder. Nat Genet 2019;51:431-44.

42 Kular L, Kular S. Epigenetics applied to psychiatry: clinical opportunities and future challenges. Psychiatry Clin Neurosci 2018;72:195-211. 